\title{
A COMMENT ON CERTAIN $p$-SHIFT ALGEBRAS
}

\author{
P. J. STACEY
}

(Received 20 March 1989)

Communicated by I. Raeburn

\begin{abstract}
Let $G=\bigoplus_{i=0}^{\infty} \mathbb{Z}_{p}$, where $p$ is a prime, let $s$ be the shift mapping the $i$ th summand of $G$ to the $(i+1)$ st and let $\omega$ be a 2 cocycle on $G$ with values in $S^{1}$, for which $\omega(s(g), s(h))=\omega(g, h)$. If $\omega\left(e_{j}, e_{k}\right)=\omega\left(e_{k}, e_{j}\right)$ whenever $|j-k|$ is sufficiently large, where $e_{i}$ is the generator of the $i$ th summand of $G$, then it is shown that the twisted group $C^{*}$-algebra $C^{*}(G, \omega)$ is isomorphic to the UHF algebra $\operatorname{UHF}\left(p^{\infty}\right)$. An immediate consequence, by results of Bures and Yin, is the existence of infinitely many non-conjugate shifts on $\operatorname{UHF}\left(p^{\infty}\right)$.
\end{abstract}

1980 Mathematics subject classification (Amer. Math. Soc.) (1985 Revision): 46 L 05, 46 L 40.

In his seminal study [4] of unital *-endomorphisms of $C^{*}$-algebras, Powers introduced a class of $A F$-algebras known as binary shift algebras which were subsequently shown in [1] to be twisted group $C^{*}$-algebras. More precisely, if $\omega$ is a multiplier on a discrete group $G$ (i.e. a 2-cocycle of $G$ with values in the circle $S^{1}$ ) then the (reduced) twisted group $C^{*}$-algebra $C^{*}(G, \omega)$ of $G$ is the $C^{*}$-algebra generated by the left regular projective representation on $l^{2}(G)$. A shift on $G$ is an endomorphism for which $\bigcap_{k=1}^{\infty} s^{k}(G)=\{e\}$, where $e$ is the identity of $G$, and, when the multiplier $\omega$ is compatible with $s$ in the sense that $\omega(s(g), s(h))=\omega(g, h)$ for all $h, g \in G$, then $C^{*}(G, \omega)$ is known as a group shift algebra. The binary shift algebras introduced by Powers are the group shift algebras for which $G$ is equal to an infinite direct sum $\bigoplus_{i=0}^{\infty} \mathbb{Z}_{2}$ of copies of $\mathbb{Z}_{2}$ and $s$ is the shift mapping the $i$ th summand of $G$ to the $(i+1)$ st summand. If, instead, $G$ is equal to an infinite direct sum $\bigoplus_{i=0}^{\infty} \mathbb{Z}_{p}$ of copies of $\mathbb{Z}_{p}$, where $p \in \mathbb{N}$, then $C^{*}(G, \omega)$ is known as

(C) 1990 Australian Mathematical Society $0263-6115 / 90 \$ A 2.00+0.00$ 
a $p$-shift algebra. Each $p$-shift algebra is approximately finite dimensional, with the approximating finite dimensional subalgebras being given by $A_{n}=$ $C^{*}\left(G_{n}, \omega\right)$, where $G_{n}=\bigoplus_{i=0}^{n} \mathbb{Z}_{p}$

It is shown in [1] that every shift-invariant multiplier $\omega$ on $G=\bigoplus_{i=0}^{\infty} \mathbb{Z}_{p}$ is specified by an infinite sequence $\{a(n): n \in \mathbb{Z}\}$ of elements of $\mathbb{Z}_{p}$ with $a(-n)=-a(n)$ for each $n \in \mathbb{Z}$; the sequence is determined by $\rho\left(e_{j}, e_{k}\right)=$ $\gamma^{a(k-j)}$, where $\gamma=\exp (2 \pi i / p), e_{i}$ is the element of $G$ corresponding to the generator 1 of the $i$ th summand $\mathbb{Z}_{p}$ and $\rho$ is the bicharacter of $G$ associated with $\omega$, defined by $\rho(g, h)=\omega(g, h) \overline{\omega(h, g)}$. Using a technique from [2] it will be shown in this note that when $p$ is a prime and $\omega$ is specified by a sequence $\{a(n): n \in \mathbb{Z}\}$ with finite support, then the $p$-shift algebra $C^{*}(G, \omega)$ is isomorphic to the $\operatorname{UHF}$ algebra $\operatorname{UHF}\left(p^{\infty}\right)$. An immediate consequence, by results from [1], is the existence of infinitely many nonconjugate shifts on the UHF algebra $\operatorname{UHF}\left(p^{\infty}\right)$.

The key lemma is based directly on [2, Lemma 2.3].

Lemma. Let $p$ be a prime and let $f(t) \in \mathbb{Z}_{p}[t]$ with $f(t)=\sum_{i=0}^{n} x(i) t^{i}$ and $x(0)=-x(n) \neq 0$. Then there exist $g(t) \in \mathbb{Z}_{p}[t]$ and $m \in \mathbb{N}$ such that $g(t) f(t)=x(n)\left(t^{m}-1\right)$.

Proof. Since $x(n) \neq 0$ we can replace $f(t)$ by $x(n)^{-1} f(t)$ and hence assume that $x(n)=1$. We inductively define $s_{k} \in \mathbb{Z}_{p}$ and $p_{k}(t) \in \mathbb{Z}_{p}[t]$ by $s_{0}=0, p_{0}(t)=f(t)$ and, for $k \geq 1, s_{k}=s_{k-1}+i_{k}$ and $p_{k}(t)=$ $x_{k-1}\left(i_{k}\right) t^{s_{k}} p_{0}(t)+p_{k-1}(t)$, where $p_{k-1}(t)=\left(\sum_{i=1}^{n} x_{k-1}(i) t^{i+s_{k-1}}\right)-1$ for some $x_{k-1}(1), \ldots, x_{k-1}(n)$ with $x_{k-1}(n) \neq 0$ and where $i_{k}=\min \{i \geq$ $\left.1: x_{k-1}(i) \neq 0\right\}$. This definition relies on $p_{k-1}(t)$ being of the stated form; to establish this inductively assume that $p_{k-1}$ is as claimed and note that the lowest non-constant term in $p_{k-1}(t)$ is $x_{k-1}\left(i_{k}\right) t^{i_{k}+s_{k-1}}=x_{k-1}\left(i_{k}\right) t^{s_{k}}$, so that the degrees of the non-constant terms of $p_{k}(t)$ lie between $s_{k}+1$ and $s_{k}+n$. Also the coefficient $x_{k}(n)$ of $t^{n+s_{k}}$ is equal to $x_{k-1}\left(i_{k}\right)$, which is non-zero by the definition of $i_{k}$. Hence the definition is consistent.

There are only finitely many elements in $\left(\mathbb{Z}_{p}\right)^{n}$ so there exist $k, l$ with $k<l$ and $x_{k}(i)=x_{l}(i)$ for each $1 \leq i \leq n$. Then $p_{l}(t)-p_{k}(t) t^{s_{l}-s_{k}}=$ $t^{s_{1}-s_{k}}-1$. This establishes the required result since, by construction, both $p_{l}(t)$ and $p_{k}(t)$ are multiples of $p_{0}(t)=f(t)$.

THEOREM. Let $p$ be a prime and let $\omega$ be a shift-compatible multiplier on $\bigoplus_{m=0}^{\infty} \mathbb{Z}_{p}$ specified by a sequence $\{a(n): n \in \mathbb{Z}\}$ for which $a(d) \neq 0$ and $a(n)=0$ for $|n|>d$. Then $C^{*}(G, \omega)$ is isomorphic to $\operatorname{UHF}\left(p^{\infty}\right)$. 
Proof. It suffices to prove that an infinite number of the finite-dimensional subalgebras $A_{n}=C^{*}\left(G_{n}, \omega\right)$ have trivial centre since then, noting that $\left\{\delta_{g}: g \in G_{n}\right\}$ is a vector space basis for $A_{n}$ with $p^{n+1}$ elements, $A_{n}$ is isomorphic to the full $p^{(n+1) / 2} \times p^{(n+1) / 2}$ matrix algebra. Thus, on passing to a subsequence, $C^{*}(G, \omega)$ is the inductive limit under unital isomorphisms of a sequence of increasing $p$-power matrix algebras.

It is well-known (as described in [3, Theorem 3.6.3]) that the dimension of the centre of $A_{n}$ is equal to the cardinality of $H_{n}=\left\{h \in G_{n}: \rho(g, h)=1\right.$ for all $\left.g \in G_{n}\right\}$, so it suffices to prove that $H_{n}$ is trivial for infinitely many $n$. Let $f(t)=\sum_{i=0}^{2 d} a(i-d) t^{i}$, let $m$ be as in the lemma, let $k \in \mathbb{N}$ and let $h=\sum_{i=0}^{k m-1} h(i) e_{i}$ belong to $H_{k m-1}$. Then, from the definition of $H_{k m-1}$ applied to $g=e_{j}$,

$$
\sum_{i=-d}^{d} h(i+j) a(i)=0 \quad \text { in } \mathbb{Z}_{p} \text { for each } 0 \leq j \leq k m-1,
$$

where $h(r)$ is defined to be zero if $r>k m-1$ or $r<0$.

By the lemma there exists a polynomial $g(t)=\sum_{i=0}^{m-2 d} b(i) t^{i}$ such that

$$
f(t) g(t)=\left(\sum_{i=0}^{2 d} a(i-d) t^{i}\right)\left(\sum_{i=0}^{m-2 d} b(i) t^{i}\right)=a(d)\left(t^{m}-1\right)
$$

Hence, equating coefficients of $t^{r}$, we obtain

$$
\sum_{i=-d}^{d} b(r-d-i) a(i)= \begin{cases}0 & \text { if } 1 \leq r \leq m-1, \\ a(d) & \text { if } r=m, \\ a(-d) & \text { if } r=0\end{cases}
$$

(where $b(j)$ is taken to be zero if $j<0$ and if $j>m-2 d$ ).

For each $0 \leq s \leq(k-1) m+2 d-1$, equations (1) yield

$$
\begin{aligned}
0 & =\sum_{j=s}^{s+m-2 d} b(j-s) \sum_{i=-d}^{d} h(i+j) a(i) \\
& =\sum_{r=0}^{m} h(r-d+s) \sum_{i=-d}^{d} b(r-d-i) a(i)
\end{aligned}
$$

and then equations (2) yield

$$
0=[h(m-d+s)-h(s-d)] a(d) .
$$

Hence $h(s-d)=h(m+s-d)$ for each $0 \leq s \leq(k-1) m+2 d-1$ and thus, in particular, $h(k m-q)=h((k-1) m-q)=\cdots=h(-q)=0$ for each $1 \leq q \leq d$. Therefore $h(t)=0$ for each $t \geq k m-d$ and then 
successive consideration of equation (1) for $j=k m-1, k m-2, \ldots, d$ yields $h(k m-d-1)=0, h(k m-d-2)=0, \ldots, h(0)=0$. Hence $h=\sum_{i=0}^{k m-1} h(i) e_{i}=0$ and so $H_{k m-1}=\{0\}$ for each $k \in \mathbb{N}$, as required.

COROLlary. The UHF algebra $\operatorname{UHF}\left(p^{\infty}\right)$ admits infinitely many nonconjugate shifts.

Proof. Recall that $C^{*}(G, \omega)$ is the $C^{*}$-algebra on $l^{2}(G)$ generated by the left regular projective representation of $G$. From [1, Proposition 1.1] any shift of $C^{*}(G, \omega)$ extends to a shift of the weak closure $W^{*}(G, \omega)$. If $G=\bigoplus_{i=0}^{\infty} \mathbb{Z}_{p}$ and $\omega$ is described by a sequence with finite support, so that $C^{*}(G, \omega)$ is isomorphic to $\operatorname{UHF}\left(p^{\infty}\right)$, then, by [1, Proposition 1.5], $W^{*}(G, \omega)$ is the hyperfinite type $\mathrm{II}_{1}$ factor. Furthermore, since the left projective representation of $G$ is cyclic with cyclic vector $\delta_{0}$ and since the corresponding linear functional on $\operatorname{UHF}\left(p^{\infty}\right)=C^{*}(G, \omega)$ is the unique trace, $W^{*}(G, \omega)$ can be identified with the closure of $\operatorname{UHF}\left(p^{\infty}\right)$ in the GNS representation associated with the trace. Hence, if two shifts of $\operatorname{UHF}\left(p^{\infty}\right)$ are conjugate then the automorphism implementing the conjugacy extends to an automorphism of $W^{*}(G, \omega)$, which will implement a conjugacy between the extended shifts. Therefore, by [1, Proposition 4.4], there exists an integer $m$ such that $a(j)=m^{2} b(j)$ for all $j \in \mathbb{Z}$, where $\{a(j): j \in \mathbb{Z}\}$ and $\{b(j): j \in \mathbb{Z}\}$ are the sequences associated with the two shifts.

By the theorem each sequence with finite support yields a shift on $\operatorname{UHF}\left(p^{\infty}\right)$, from which the result follows.

\title{
References
}

[1] D. Bures, and H. S. Yin, 'Shifts on the hyperfinite factor of type II , J. Operator Theory 20 (1988), 91-106.

[2] M. Enomoto, M. Nagisa, Y. Watatani and H. Yoshida, 'Relative commutant algebras of Powers' binary shifts on the hyperfinite II 1 factor,' preprint, 1988.

[3] G. Karpilovsky, Projective representation of finite groups, (Marcel Dekker, 1985).

[4] R. T. Powers, 'An index theory for semigroups of *-endomorphisms of $B(H)$ and type II 1 factors,' Canad. J. Math. 40 (1988), 86-114.

\author{
La Trobe University \\ Bundoora \\ Victoria 3083 \\ Australia
}

